\title{
On the design of electromagnetic actuators with increased vibration resistance and low vibration activity for the mechatronic devices of autonomous transport systems
}

\author{
Irina A. Bolshenko ${ }^{1}$, Alexandr V. Pavlenko ${ }^{1, *}$, Konstantin R. Gil'miyarov ${ }^{2}$, and Andrey A. \\ Gummel' $^{1}$ \\ ${ }^{1}$ Department of Electromechanics and Electric Apparatus, Novocherkassk Platov South-Russian State \\ Polytechnic University (NPI), 346400 Novocherkassk, Russian Federation \\ ${ }^{2}$ Rostov-on-Don Research Institute of Radiocommunications, 344038 Rostov-on-Don, Russian \\ Federation
}

\begin{abstract}
The questions of complex designing of electromagnetic actuators, with increased resistance to external mechanical influences and low vibration activity, focused on the use of transport systems in mechatronic devices are considered. As a determining direction for creating actuators with increased vibration resistance and low vibration activity, the direction of realization of electromagnetic mechanisms with an adaptive control system that controls external mechanical influences and vibronoise parameters of the device is proposed. A complex mathematical model describing electromechanical processes in an electromagnetic actuator, that takes into account the influence of external factors, is considered. Based on the studies, three adaptive control algorithms are proposed, differing in the way they determine the current value of the speed and position of the lifter. The proposed algorithms are implemented experimentally, have shown good stability and efficiency.
\end{abstract}

\section{Introduction}

When designing the mechatronic modules of power-plants of autonomous transport systems, including robotic underwater and surface devices, special attention is paid to solving two problems. The first of them is related to the influence of external mechanical influences on the parameters and characteristics of electromagnetic actuators and other electromechanical converters on the performance of the system as a whole. The second problem arises in connection with the increased noise level produced by electromechanical converters and, in many ways, determines and limits the possibility of using the equipment. These problems are particularly acute in the automotive and shipbuilding industries. The autonomous transport systems created in these industries are subject to high requirements not only in terms of comfort, but also the possibility of detecting them at an increased level

\footnotetext{
* Corresponding author: niiem.srstu@gmail.com
} 
of noise. The noise and vibration of the devices form the underwater noise of ships and submarines of the Navy, worsening their secrecy. The works on creating electromechanical converters with increased vibration resistance and low vibration activity is being carried out in many countries around the world in two developing directions: the development of new actuator designs and the creation of new design methods that take into account the conditions of their operation. Some high-tech designs of electromechanical converters created by scientists and engineers from Germany, the United States, Korea, China and other countries are known [1-4]. Basically, these are the actuators of various automotive mechatronic devices and systems. The second direction of work is presented to a lesser degree and, mainly, by German scientists and engineers [5-9]. In many publications related to the development of design methods, the damping effect of eddy currents in solid magnetic conductors and the nonlinearity of the magnetization characteristics of steels are to some extent taken into account. All studies differ in the formulation of the problem and in the hypotheses made in its solution. In studies [10-14] questions of the design of electromagnetic actuators of valves of air and gas exchange systems and fuel supply of internal combustion engines of vehicles more exposed to external vibrations and, on the other hand, during cyclic operation, they are sources of acoustic noise. Mathematical models and algorithms for mathematical modeling of electromagnetic actuators, methods and algorithms of adaptive control of electromagnetic mechanisms are proposed in the articles. The influence of external mechanical influencing factors and the vibration activity of devices during design are practically not taken into account and determined experimentally.

\section{Adoptive control algorithms}

In this study, the direction of realization of electromagnetic mechanisms with an adaptive control system that controls the vibration parameters of the device is proposed as the determining direction for the creation of actuators with low vibration activity. The basis of the technique is the synthesis of signals controlling the electromagnetic mechanism, providing low vibration activity and stability of the dynamic parameters of devices at given vibration levels and other types of disturbing effects in real time. To control valves in fuel supply systems and air and gas exchange of power plants of autonomous transport systems [10], which are an interconnected electromechanical system consisting of two electromagnets and armature general, with two spring-loaded on both sides. It is advisable to consider the main ratios of the design methodology on the example of such an actuator. In such a mechanism, the electromagnetic force is used only to compensate the resistance forces and to retain the lifter in the extreme positions.

In this case, the movement of the lifter is due to the energy stored in the springs. To reduce the power consumption at low switching frequencies, the design is supplemented by permanent magnets (Fig. 1) [15]. One of the main tasks in the design of the actuator is to ensure shock-free operation and, accordingly, to reduce acoustic noise. The solution of this problem requires an integrated approach to the design of the actuator structure in conjunction with the adaptive control system. To form the control actions, the adaptive control system needs information about the current state of the process, which can be obtained by calculation. Therefore, the main design stage after choosing the configuration of the magnetic system and determining the geometric dimensions is mathematical modeling, determination of characteristics and parameters of the actuator, carried out in real time. A complex mathematical model is formed on the basis of the equalization of electrical circuits, the kinematics of the mechanism, the use of relationships previously obtained in the calculation of the electromagnetic field. The kinematic scheme of the electromagnetic 
actuator (EMA), focused on control of the valve of the gas distribution mechanism (GDM), is presented in Fig.2.

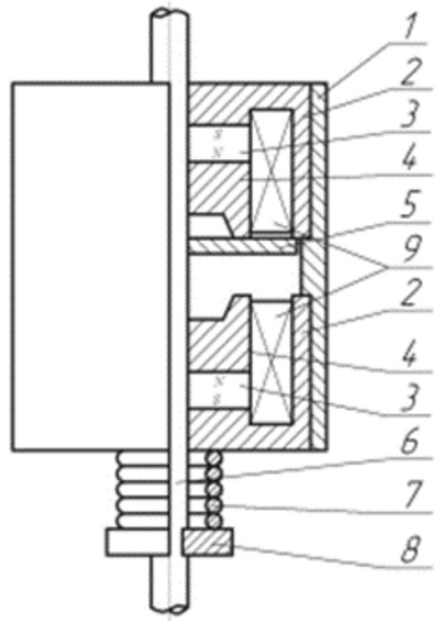

Fig. 1. Drawing of design of electromagnet: 1 body; 2 - magnetic circuit; 3 - permanent magnet; 4 - effective air gap; 5 - lifter; 6 - rod; 7 - spring; 8 - lock ring; 9 - control winding.

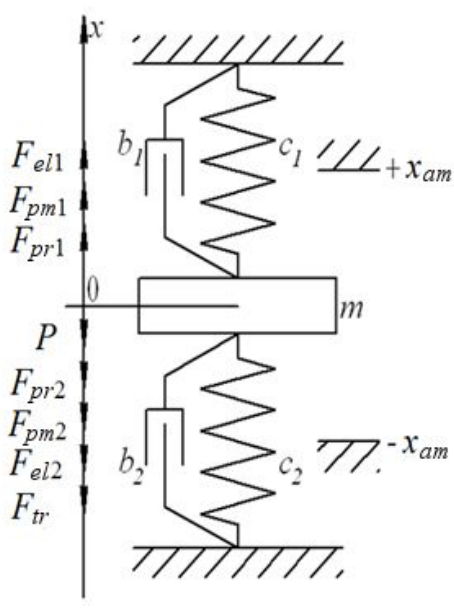

Fig. 2. Kinematic scheme of actuator.

Electromechanical processes in the actuator are described by a system of equations:

$$
\left\{\begin{array}{l}
m a=F_{e l 1}+F_{p m 1}-F_{e l 2}-F_{p m 2}+F_{p r 1}-F_{p r 2}-F_{t r}-P-F_{u d}, \\
a=\frac{\mathrm{d} v}{\mathrm{~d} t}, v=\frac{\mathrm{d} x}{\mathrm{~d} t} \\
F_{e l 1}=f_{1}\left(i_{1}, x\right), F_{e l 2}=f_{2}\left(i_{2}, x\right), \\
F_{p m 1}=f_{3}(x), F_{p m 2}=f_{4}(x), \\
i_{1}=f_{5}\left(\Psi_{1}, x\right), i_{2}=f_{6}\left(\Psi_{2}, x\right), \\
\Psi_{1}=\int\left(U_{1}-i_{1} \cdot R_{1}\right) \mathrm{d} t, \\
\Psi_{2}=\int\left(U_{2}-i_{2} \cdot R_{2}\right) \mathrm{d} t, \\
F_{p r 1}=c_{1} \cdot x, \\
F_{p r 2}=c_{2} \cdot x, \\
F_{t r}=b \cdot v, \\
P=m g, \\
F_{u d}=\left\{\begin{array}{l}
\frac{m \cdot v_{u d}}{t_{u d}} \cdot(1+k), a t|x|=x_{a m} \\
0, a t-x_{a m}<x<x_{a m}
\end{array}\right.
\end{array}\right.
$$

Here: $m$ - total mass of moving parts of the actuator; $a, v, x$-acceleration, speed and lifter stroke; $F_{e l 1}$ and $F_{e l 2}$ - forces generated by electromagnets; $F_{p m 1}$ and $F_{p m 2}$ - forces created by permanent magnets; $U, R_{1}, R_{2}, i_{1}, i_{2}$ - supply voltage, active resistances and currents in the windings of electromagnets; $\Psi_{1}$ and $\Psi_{2}$ - flux linkages of windings; $F_{p r 1}$ and 
$F_{p r 2}-$ spring forces; $c_{1}$ and $c_{2}-$ spring rate; $F_{t r}$ - viscous friction force; $b$ - coefficient of viscous friction; $P$ - force of gravity; $g$ - acceleration of gravity; $F_{u d}$ - force that characterizes the impact; $t_{u d}$ - duration of impact; $v_{u d}$ - speed of lifter at the moment of impact; $k$ - recovery coefficient; $x_{a m}$ - extreme position of lifter. The correspondences $\Psi=f(i, x)$ and $F_{e l}=f(i, x)$ are determined from the results of the magnetic field calculation and are subsequently used in the complex model to determine the dynamic characteristics of the resonant electromagnetic mechanism. For convenience of calculations, the correspondence of the flux linkage with the current and gap $\Psi=f(i, x)$ is converted into a current correspondence with flux linkage and gap $i=f(\Psi, x)$.

The system of equations describing the influence of external factors affecting the actuator that controls the GDM valve, can be represented in the form:

$$
\left\{\begin{array}{l}
c=E(T) \cdot \frac{S_{0}}{l} \\
b=\mu_{0} \cdot e^{a_{\mu}\left(T-T_{0}\right)} \cdot \frac{S}{h_{S}} \\
R_{1}=R_{0} \cdot\left(1+\alpha_{T} \cdot T_{1}\right), \\
R_{2}=R_{0} \cdot\left(1+\alpha_{T} \cdot T_{2}\right) \\
F_{v i b}=m \cdot a_{v i b}, \\
a_{v i b}=A_{v i b} \cdot \sin \left(\omega_{v i b} \cdot t+\varphi_{v i b}\right) \\
F_{g a s}=\Delta p \cdot S_{k l}, \\
\Delta p=\xi \frac{\rho \cdot u^{2}}{2}, \\
\xi=2,7-\frac{0,8}{x / d_{k}}+\frac{0,14}{\left(x / d_{k}\right)^{2}}
\end{array}\right.
$$

Here: $R_{0}$ - active resistance of windings at $0^{\circ} \mathrm{C} ; T_{1}$ and $T_{2}$ - temperatures of windings; $\mathrm{E}$ - elasticity modulus; $S_{0}$ and $l$ - cross-sectional area of wire and length of spring; $\mu$ and $\mu_{0}$ - values of dynamic coefficient of viscosity at temperature $t$ and $t_{0}$ degrees; $\alpha_{\mu}$ - exponent, depending on the kind of fluid; $a_{v i b}, A_{v i b}, \omega_{v i b}, \varphi_{v i b}-$ acceleration, range, frequency and phase of source of vibration; $\Delta p$ - pressure difference on valve; $S_{k l}$ - cross-sectional area of valve; $\xi$ - coefficient of local resistance; $\rho$ - gas density; $u$ - rate of outflow of gases in valve, $d_{k}-$ the diameter of the valve.

The joint solution of the above-mentioned systems of equations allows one to evaluate the effect of changes in ambient temperature, susceptibility to vibrations, and the effect of the pressure difference acting on the GDM valve, on the dynamic characteristics of the actuator. In this case, the speed of the moving element, the impact force and the level of acoustic noise produced can be determined, respectively. The model is implemented in the on-board computer in conjunction with a control algorithm that provides shock-free operation and specified dynamic characteristics of the EMA.

The authors of this article proposed three adaptive control algorithms [16-18], differing in the way of determining the current value of speed and position of the lifter.

The first method and the control algorithm [16] are based on the indirect determination of the spatial position and speed of the lifter at the moment of approach to the pole in real time mode by the nature of the current variation in the windings and the voltage on them. The control method is based on the fact that when the magnitude of the magnetic flux 
changes in the magnetic circuit during the movement of the lifter, an EMF of self-induction occurs in the winding of the electromagnet, causing change in the current. This change represents a "failure" on the dynamic characteristic of the current at the time the lifter approaches the pole, and the larger the value, the greater the speed at the end of the lifter stroke. By controlling the value of the current "failure" in real time, it is possible to vary the lifter speed in a significant range, including at the end of its stroke, by adjusting the time parameters of the control pulses (PWM).

Another adaptive algorithm [17] is based on measuring the rate of change in the current in the EMA winding while moving the lifter. This control algorithm can also be used to construct a control system for electromagnetic actuators that provide compensation for external disturbances, such as external load variables on the actuator of the actuator, regulate the speed of the moving element during movement and at the end of the stroke, the ambient temperature. The advantage of the proposed method is to compensate slowly changing from one switching to another, external influences (ambient temperature, heating of the electromagnetic actuator elements) and accidental mechanical action on the movable actuator element (vibration, etc.) during the current switching. The method is based on the interconnection of the electrical processes taking place in the winding of the electromagnetic actuator with mechanical processes caused by the movement of the movable element of the magnetic system. When the lifter moves, the magnetic conductivity of the air gap changes and, consequently, the amount of air flow in it too, which leads to a change in the winding current at constant voltage. When moving the lifter, in the coil of the electromagnet, along with the EMF-self-induction, there is an EMF movement whose magnitude depends on the speed of the lifter of the electromagnet, leading to a change in the current, respectively, to a change in the force developed by the electromagnet. Both algorithms are implemented experimentally [11] and showed good stability and efficiency.

A promising method for controlling EMF, which provides a condition for landing the lifter with a minimum speed in the mode of switching, is that the control action is formed depending on the change in the current value of the kinetic energy of the lifter [18]. Part of the potential energy is spent on overcoming the forces of frictional resistance, gas pressure on the valve of the gas distribution mechanism, vibrations. The distribution of energy when moving the lifter will correspond to the energy distribution in the ideal (conservative) mechanical actuator system provided that the energy cost is compensated for overcoming the resistance forces $W_{d}$. In this case, the movable element of the actuator will move to the position $x_{u d}$ with the specified travel time and zero speed at the moment of valve landing. The equation of the energy balance of the mechanical system of the electromagnet can be written in the following form:

$$
W_{\Sigma}=W_{p}+W_{k}+W_{d}+W_{e l}+W_{p m} .
$$

Here $W_{p}$ - potential energy of the system; $W_{k}$ - kinetic energy; $W_{d}-$ energy spent on overcoming the forces of resistance; $W_{e l}$ and $W_{p m}$ - energies supplied by electromagnets and permanent magnets.

Compensation of energy costs to overcome the forces of resistance $W_{d}$ is possible only due to the energies $W_{e l}$ and $W_{p m}$, which are converted during the movement of the lifter into mechanical energy. Taking into account the fact that the action of permanent magnets at the stage of acceleration impedes the movement of the lifter, in order to ensure the switching of the actuator with the set operating time and the minimum speed of the lifter, it is necessary to fulfill the condition $W_{e l}=W_{d}+W_{p m}$. Thus, the essence of the proposed control method is to ensure the fulfillment of this condition by regulating the amount of energy supplied by the electromagnets. At the same time, the mechanical system achieves the properties inherent in an ideal system - providing the set operating time and the minimum landing 
speed of the lifter. The algorithm of the control method is implemented in accordance with the following sequence:

- based on the current values of the position of the lifter $x(t)$ and the speed $v(t)$, the values of the potential $W_{p}(t)$ and the kinetic energies of the system $W_{k}(t)$ are calculated.

- the required value of the kinetic energy of the lifter is calculated from the current value $W_{p}(t)$ and the maximum value of the potential energy $W_{\Sigma}(t), W_{k}{ }^{\prime}(t)=W_{\Sigma}(t)-W_{p}(t)$.

- based on the current values of the real $W_{k}(t)$ and the required $W_{k}{ }^{\prime}(t)$ kinetic energy of the lifter, actuating error $\Delta W_{k}(t)=W_{k}(t)-W_{k}(t)$ is calculated.

- the current value of the position of the lifter $x(t)$ determines the energy derivative $\Delta W_{k}(t)$ in terms of the air gap $\delta(t)$, i.e. value of the electromagnetic force $F_{e l}(t)$.

- the value of the current $i(t)$, formed in the corresponding control windings, is calculated from the value of the electromagnetic force $F_{e l}(t)$, which must be created to compensate $\Delta W_{k}(t)$.

The magnitude of the electromagnetic force $F_{e l}$, nonlinearly depends on the values of the current $i$, air gap $\delta$, eddy currents, saturation of the magnetic core steel and other factors, therefore it is rather difficult to obtain its analytical description. In connection with this, for the implementation of the control algorithm, the fuzzy logic device is used, which has proved itself in the absence of an accurate mathematical description of the control object. At the same time, the EMA control system consists of two circuits - an internal current control loop in the windings of electromagnets and an external control loop for the kinetic energy of the lifter. Current regulation in the windings of the electromagnetic actuator is carried out using various widely known control laws. Synthesis of the fuzzy model of the lifter kinetic energy regulating unit includes two stages.

At the first stage - stage of structural identification - an approximate model of the regulation unit is formed and its coarse adjustment is carried out. The fuzzy model is based on the Mamdani model, whose input variables are the magnitude of the force $F_{e l}$ and the current position of the lifter $x$, and the output variable is the current $i$ that must be formed in the windings of the electromagnets. As a method of defuzzification, the method of center of gravity is used. The range of input and output variables is divided into several terms and in accordance with the grammar of linguistic variables, the rule base is built. The stage is completed by defining the form of membership functions, which are chosen as the membership functions of the triangular form. The initial information for constructing the rule base and determining the form of the membership functions is the function $i=f\left(F_{e l}, x\right)$. At the parametric identification stage, to increase the accuracy, the fuzzy model of the kinetic energy regulation unit of lifter is trained, i.e. its parameters are selected by iterations in order to minimize the deviation of the logical output results from the initial data. The teaching of the fuzzy Mamdani model is a task of nonlinear optimization, which can be solved by using various optimization algorithms.

\section{Conclusions}

The effectiveness of the proposed methods and control algorithms in conjunction with the complex mathematical model is confirmed experimentally [19]. The test results showed that the application of the proposed control method makes it possible to provide a final valve landing speed of less than $0.4 \mathrm{~m} / \mathrm{s}$ and a switching time of about $6 \mathrm{~ms}$ under the influence of external disturbances. The proposed tools can be used in designing electromagnetic actuators with increased vibration resistance and low vibration activity.

The research was performed funded with the Russian Science Foundation grant (project № 17-7910055). 


\section{References}

1. M. Bösing, Acoustic modeling of electrical drives. Noise and vibration synthesis based on force response superposition, Dissertation to obtain the degree of a Doctor-Engineer (RWTH AachenUniversity, 2013)

2. J. Melbert, R. Uhlenbrock, IEEE/ASME Trans. Mech., 3 (2003)

3. R. Subashraj, S. Prabhu, A. Manikandan, N. Lenin, V. Chandrasekar, R. Arumugam, IJIRD, 3, 78 (2014)

4. J. Blum, J. Merwerth, H.G. Herzog, IEEE/4th Int. Electr. Drives Product. Conf. (EDPC), 14833353 (2014)

5. S. Parrang, J. Ojeda, S. Khelladi, M. Gabsi, IEEE/7th IET Int. Conf. Power Electron. Mach. Drives (PEMD 2014), 14250975 (2014)

6. J. Fiedler, Design of low-noise switched reluctance drives (Germany: Shaker Verlag $\mathrm{GmbH}, 2007$ )

7. J. Le Besnerais, IEEE/XXII Int. Conf. Electr. Mach. (ICEM), 16444183 (2016)

8. E. Devillers, M. Hecquet, J. Le Besnerais, IEEE/12th Int. Conf. Ecol. Veh. Renew. Energies (EVER), 16930172 (2017)

9. M. Régniez, P. Bonneel, J. Le Besnerais, 24th Int. Congr. Sound Vib. (ICSV24), 6, 4472 (2017)

10. B. Özdalyan, O. Doğan, J. Zhejiang Univ. Sci. A, 11, 106 (2010)

11. A. Pavlenko, D. Batishchev, A. Gummel', I. Bolshenko, Russ. Electromech., 60 (3), 17 (2017)

12. A. Pavlenko, K. Gil'miyarov, Univ. News. North-Caucas. Reg. Tech. Sci. Series, 128 (3), 257 (2012)

13. Y. Qiu, D. Perreault, J. Kassakian, T. Keim, IEEE/5th IET Int. Conf. Power Electron. Mach. Drives (PEMD 2010), 1 (2010)

14. Y. Qiu, D. Perreault, T. Keim, J. Kassakian, IEEE/ASME Trans. Mech., 17, 1098 (2012)

15. Patent RU 2242816 C2 Fast-acting polarized armored-type electromagnet with independent polarization fluxes (2002)

16. A. Pavlenko, A. Gummel', D. Batishchev, E. Baumbach, Russ. Electr. Engin., 87, 189 (2016)

17. A. Pavlenko, A. Gummel, I. Bolshenko, Procedia Engineering, 129, 571 (2015)

18. A. Pavlenko, K. Gilmiyarov, Univ. News. North-Caucas. Reg. Tech. Sci. Series, 1, 46 (2012)

19. K. Gil'miyarov, Method and device for controlling the mechatronic drive of the valve of the gas-distribution mechanism of the internal combustion engine, Dissertation to obtain the degree of a PhD in Technical Sciences (SRSTU(NPI), Novocherkassk, 2012) 